\title{
4. \\ Note, relative à un mémoire de Mr. Richelot sur quelques intégrales définies.
}

(Par Mr. Chr. Jïrgensen de Copenhague.)

Dans le Tome XXI. pag. 293 de ce journal se trouve un mémoire de Mr. Richelot sur quelques intégrales définies, dont la somme peut s'exprimer au moyen de la quadrature et de la division du cercle. Je vais faire voir, que les théorèmes fort interessants auxquels l'auteur y est parvenu, et d'autres semblables peuvent être démontrés d'une manière un peu plus simple, ou plutôt qu'ils sont tous contenus comme cas particuliers dans la formule générale pour la décomposition des fonctions de la forme

$$
\boldsymbol{F} \boldsymbol{b}=\frac{B}{\left(b-a_{1}\right)^{1-\mu_{1}}\left(b-a^{2}\right)^{1-\mu_{2}} \ldots\left(b-a_{n}\right)^{1-\mu_{n}}},
$$

$\boldsymbol{B}$ étant une fonction entière de $b$ et $\mu_{1}, \mu_{2}, \ldots \mu_{n}$ les quantités comprises entre zéro et l'unité, formule que j’ai donnée vol. XIX. de ce journal. En effet, si l'on transcrit en quadratures définies la dernière formule de ce mémoire (vol. XIX. pag. 90) on aura, en mettant $b$ au lieu de $x$,

$$
\boldsymbol{F} \boldsymbol{b}={\underset{i=1}{i=1} \frac{\sin \mu_{i} \pi}{(-1)^{\mu_{i} \pi}}}_{0}^{1} \frac{F\left(\frac{a_{i}}{\theta}\right)}{b \theta-a_{i}} \cdot \frac{a_{i} \partial 0}{\theta}+\boldsymbol{H}\left(\frac{F t}{t-b}\right),
$$

sous condition que $b<a_{i}$ pour toutes les valeurs de $i$, et que $\left(\frac{F t}{t-b}\right)$ puisse être développé suivant les puissances entières déscendantes de $t$, ce qui a lieu lorsque $\mu_{1}+\mu_{2}+\ldots .+\mu_{n}$ est un nombre entier. Si l'on fait dans cette formule $\frac{a_{i}}{\theta}=x$, on trouve en renversant les limites

$$
\boldsymbol{F} b=\boldsymbol{S} \frac{\sin \mu_{i} \pi}{(-1)^{\mu_{i}}} \int_{a_{i}}^{a_{i}} \frac{F x}{b-x} \partial x+H\left(\frac{F t}{t-b}\right) .
$$

Cette formule est même un peu plus générale que la précédente, en ce qu'on peut prendre la quantité $\frac{a_{i}}{0}$ en plus ou en moins pour éviter le passage par l'infini ainsi que la condition $b \ll a_{i}$. Cela tient à ce qu'on ne peut pas faire varier $\frac{a_{i}}{t}$ continuellement depuis zéro jusqu'à l'iufini de signe contraire en faisant varier $\theta$ depuis l'unité jusqu'à zéro. 
Il serait facile de présenter la démonstration de ces formules indèpendamment de la considération des intégrales à indices fractionnaires et de la même manière du reste, dont nous nous sommes servi à l'endroit cité, mais on y parvieudra de la manière bien plus simple encore, que Mr. Ramtes, professeur à l'université de Copenhague, a bien voulu me communiquer, et que voici.

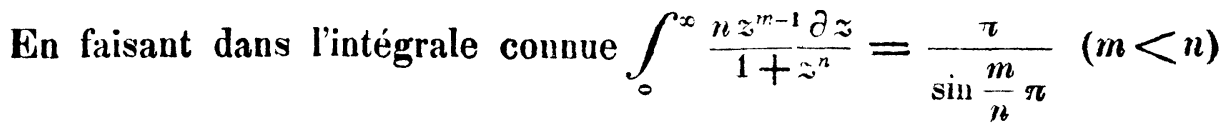

(Lacroix Traité du calc. diff. et int. III. pag. 417 et suiv.) $\approx^{n}=y$, et écrivant ensuite $\mu_{0}$ au lieu de $\frac{m}{r}$, il vient

$$
\int_{0}^{\infty} \frac{\partial y}{(1+y) y^{1-\mu}}=\frac{\pi}{\sin \mu \pi}
$$

Supposant $y=\frac{x-a}{a-b}$, on trouve

$$
\frac{\sin \mu \pi}{(-1)^{\mu} \pi} \int_{a}^{ \pm \infty} \frac{\partial x}{(b-x)(x-a)^{1-\mu}}=\frac{1}{(b-a)^{1-\mu}},
$$

où l'on doit prendre le signe + si $b<a$ et le signe - si $b>a$. Maiıtenaut on a la formule connue

$$
f b=\frac{b}{\left(b-a_{1}\right)(b-a) \ldots\left(b-a_{n}\right)}=\boldsymbol{S} \frac{\varphi_{i} a_{i}}{b-a_{i}}+\boldsymbol{H}\left(\frac{f t}{t-b}\right),
$$

$\varphi_{i} x$ étant $=\left(x-a_{i}\right) f x$ et $\boldsymbol{H}$ le coëfficient de $\frac{1}{t}$. Mettant dans cette formule $x$ au lieu de $a_{i}$, multipliant $\operatorname{par} \frac{\sin \mu_{i} \pi}{(-1)^{\mu_{i}}} \cdot \frac{\partial x}{\left(x-a_{i}\right)^{1-\mu_{i}}}$, intégrant depuis $x=a_{i}$ jusqu’à $x= \pm \infty$, selon que $b<a_{i}$ ou $b>a_{i}$ et répétant ceci pour toutes les valeurs de $i$ depuis 1 jusqu'à $n$, on trouve le théorème suivant.

Lorsque $a_{1}>a_{2}>a_{3} \ldots>a_{n}$ et $a_{p}>b>a_{p+1}$ on a

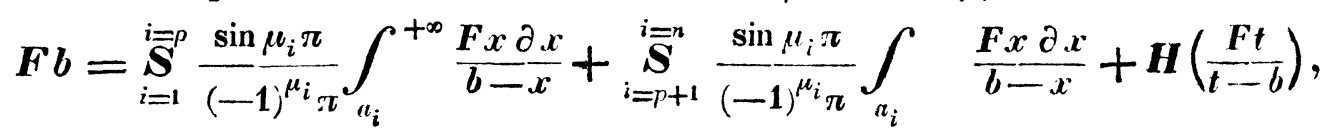

$\boldsymbol{F} b$ désignant, comme ci-dessus, la fonction

$$
\frac{B}{\left(b-a_{1}\right)^{1-\mu_{1}}\left(b-a_{2}\right)^{1-\mu_{2}} \ldots\left(b-a_{n}\right)^{1-\mu_{n}}}
$$

11 est très facile de déduire delà tous les théorèmes fondanentaux de Mr. Richelot. Si l'on veut avoir le théorème $5^{\text {ine }}$ page 314 et 315 par exemple, on fera $B=f b, \mu_{1}=\mu, \mu_{2}=1-\mu_{1}, \mu_{3}=\mu, \mu_{4}=1-\mu$, et ainsi de suite jusqu'à $\mu_{2 k-1}=\mu, \mu_{2 k}=1-\mu$, de sorte que deux termes consé- 
cutifs sous le signe $\boldsymbol{S}$ deviennent, en supposant que $b$ ne soit pas compris entre les limites $a_{2 m-1}$ et $a_{2 m}$ et que $a_{2 m-1}>a_{2 m}$,

$$
\begin{aligned}
& \frac{\sin \mu \pi}{(-1)^{\mu} \pi} \int_{a_{2 m-1}}^{ \pm \infty} \frac{f x \partial x}{(b-x)\left(x-a_{1}\right)^{1-\mu}\left(x-a_{2}\right)^{\mu} \ldots\left(x-a_{2}\right)^{\mu}}+\frac{\sin (1-\mu) \pi}{(-1)^{1-\mu} \pi} \int_{a_{2 m}}^{ \pm \infty} \frac{f x \partial x}{(b-x)\left(x-a_{1}\right)^{1-\mu}\left(x-a_{2}\right)^{\mu} \ldots\left(x-a_{2 k}\right)^{\mu}} \\
& \quad=-\frac{\sin \mu \pi}{\pi} \int_{a_{2 m}}^{a_{2 m-1}} \frac{f x \partial x}{(x-b)\left(a_{1}-x\right)^{1-\mu}\left(x-a_{2}\right)^{\mu} \ldots\left(x-a_{2 k}\right)^{\mu}}=+\frac{\sin \mu \pi}{\pi} \int_{a_{2 m}}^{a_{2 m-1}} \frac{X f x \partial x}{x-b}
\end{aligned}
$$

suivant la notation dans le mémoire cité page 315. (Par faute d'impression il y a $x-a_{1}$ sous le radical au lieu de $a_{1}-x$; voyez page 313). On tronve ainsi

$$
\int_{a_{2}}^{a_{1}} \frac{X f x \partial x}{x-b}+\int_{a_{4}}^{a_{2}} \frac{X f x \partial x}{x-b}+\ldots+\int_{a_{2 k}}^{2 k-1} \frac{X f x \partial x}{x-b}=\frac{\pi}{\sin \mu \pi}\left\{F b-H\left(\frac{F t}{t-b}\right)\right\},
$$

ce qui est le $5^{\text {me }}$ théorème.

Pour avoir le $\boldsymbol{7}^{\text {ine }}$ théorème page 323, on fera $B=f b, \mu_{1}=\mu$, $\mu_{2}=1-\mu, \mu_{3}=1-\mu, \mu_{1}=\mu, \mu_{5}=\mu, \mu_{6}=1-\mu, \cdots \mu_{+k-1}=1-\mu$, $\mu_{4 k}=\mu$, de sorte que les deux premiers termes sous le signe $\boldsymbol{S}$ deviennent, en supposant que $b$ soit hors des limites $a_{1}$ et $a_{2}$ et que $a_{1}>a_{2}$,

$$
\begin{gathered}
\frac{\sin \eta^{\prime} \pi}{(-1)^{\mu} \pi} \int_{a_{1}}^{ \pm \infty} \frac{f x \partial x}{(b-x)\left(x-a_{1}\right)^{1-\mu}\left(x-a_{2}\right)^{\mu} \ldots}+\frac{\sin (1-\mu) \pi}{(-1)^{1-\mu} \pi} \int_{a_{2}}^{ \pm \infty} \frac{f x \partial x}{(b-x)\left(x-a_{1}\right)^{1-\mu}\left(x-a_{2}\right)^{\mu} \ldots} \\
\quad=-\frac{\sin \mu \pi}{\pi} \int_{a_{2}}^{a_{1}} \frac{f x \partial x}{(x-b)\left(a_{1}-x\right)^{1-\mu}\left(x-a_{2}\right)^{\mu} \ldots}=\frac{\sin \mu \pi}{\pi} \int_{a_{2}}^{a_{1}} \frac{X f x}{x-b}
\end{gathered}
$$

suivant la notation du mémoire cité page 323. Les deux termes suivants deviennent

$$
\begin{gathered}
\frac{\sin (1-\mu) \pi}{(-1)^{1-\mu} \pi} \int_{a_{3}}^{ \pm \infty} \frac{f x \partial x}{(b-x)\left(x-a_{1}\right)^{1-\mu}\left(x-a_{2}\right)^{\mu} \ldots}+\frac{\sin \mu \pi}{(-1)^{\mu} \pi} \int_{a_{4}}^{ \pm \infty} \frac{f x \partial x}{(b-x)\left(x-a_{1}\right)^{1-\mu}\left(x-a_{2}\right)^{\mu} \ldots} \\
=+\frac{\sin \mu \pi}{\pi} \int_{a_{4}}^{a_{3}} \frac{f x \partial x}{(x-b)\left(a_{1}-x\right)^{1-\mu}\left(x-a_{2}\right)^{\mu} \ldots}=\frac{\sin \mu \pi}{\pi} \int_{a_{3}}^{a_{4}} \frac{X f x \partial x}{x-b}
\end{gathered}
$$

et ainsi de suite, donc

$$
\begin{aligned}
& \int_{a_{2}}^{a_{1}} \frac{X f x \partial x}{x-b}+\int_{a_{3}}^{a_{4}} \frac{X f x \partial x}{x-b}+\ldots+\int_{a_{4 k-1}}^{a_{4 k}} \frac{X f x \partial x}{x-b}=\frac{\pi}{\sin \pi \mu}\left\{F b-H\left(\frac{F t}{t-b}\right)\right\}, \\
& \text { ce qui est le gine théoréme. }
\end{aligned}
$$

$$
27 \text { Mars } 1841 .
$$

